\title{
Application of Computer Aided Simulation and Biomechanical Analysis in Treatment of Congenital Sagittal Synostosis
}

\author{
Li Xiang ${ }^{1}$, Lu Zheng ${ }^{1}$, Li Zhicen ${ }^{1}$, Zhu Wanchun ${ }^{1}$, He Jintao ${ }^{1}$, Yu Yaxiong ${ }^{1}$, Gong Jian ${ }^{1}{ }^{*}$ \\ ${ }^{1}$ Beijing Tiantan Hospital Affiliated to Capital Medical University, Beijing 100070, China
}

\begin{abstract}
This study explores the application value of computer aided simulation and biomechanical analysis in treatment of congenital sagittal synostosis. The traditional and proposed treatment methods were evaluated by comparing the operation time, blood loss, operation cost and postoperative complications. The application of computer simulation surgery and biomechanical analysis in the treatment of children with congenital sagittal synostosis allows surgeons to be familiar with the operation process and splicing scheme in advance. In particular, computer biomechanical analysis was proposed for the first time to determine the position and quantity of the joint materials, so as to achieve operation's objectives of stability, aesthetics, expansion, significantly improve the surgical efficiency, reduce the cost and postoperative complications.
\end{abstract}

\section{INTRODUCTION}

Craniosynostosis is caused by the premature closure of sutura cranii. Craniosynostosis occurs in 1 in every 2,000 to 3,000 births [1]. There are many types of craniosynostosis, depending on the location of craniosynostosis (sagittal, coronal, frontal, and hermetic) and number of craniosynostosis. One or more craniosynostosis may results in a variety of changes in central nervous function, craniofacial morphology, and cranial volume. It has been reported that the incidence of sagittal synostosis accounts for $0.4 \%$ in newborns [2]. In the author's research center, sagittal synostosis was also dominant in the last 2 years. Therefore, this paper focuses on sagittal synostosis. Surgery to reduce intracranial pressure and correct malformation is still the main treatment of craniosynostosis.

As neurosurgeons or orthopedic surgeons continue to explore ways to treat craniosynostosis, many treatments have been developed, from simple resection of the diseased craniosynostosis in the early day to the present endoscopic surgery, spring supported craniosynostosis implantation, and helmet orthopedic surgery. Among them, reconstruction of the skull is an important treatment for craniosynostosis. However, due to the lack of specific standardized surgical procedures, there are many disadvantages in the process of reconstruction of the skull. Since there is no simulation before surgery and the operation opportunity is only once, surgeons have no clear concept of the individualized postoperative effect of patients before surgery, which makes the postoperative effect sometimes not satisfactory. In addition, the reconstruction of the skull requires the implantation of fixed objects, such as titanium sheets. Too many of these implants undoubtedly increase the patient's cost, operation time, and risk of postoperative infection.

In recent years, advances in science and technology have made it possible to simulate surgery in $3 \mathrm{D}$ computers. The 3D surgical simulation of skull reconstruction can be intuitively completed in the computer, which enables surgeons to simulate multiple surgical schemes of skull reconstruction for the same patient and ultimately select the best individualized treatment. Moreover, if the neurosurgeon already has a certain understanding of the surgery outcome before surgery, it will undoubtedly reduce the operation time, reduce the amount of blood loss, and reduce the risk of surgery for patients. At the same time, biomechanical analysis can be used to analyze the optimal placement and number of implants after skull reconstruction, so as to achieve a perfect mechanical support effect with the least implants, which will undoubtedly reduce the cost of patients, the risk of postoperative infection and the operation time. The treatment of craniosynostosis should take into account not only aesthetics, but also the parents' concern about the shape of the child's skull and the psychological problems $[3,4]$. There is no doubt that the individualized virtual reality operation will significantly improve the postoperative effect [5].

Over the past two years, the authors have been committed to find the best surgical option for clinical application based on 3D surgical simulation and biomechanical computation, especially for the treatment of sagittal synostosis. The advantages and disadvantages of the new treatment method are analyzed by retrospective analysis of the clinical data of patients with traditional treatment, and the procedures of the new treatment method are elaborated. 


\section{Material and method}

2.1 Individual patient data from 2014 to 2016, a total of 18 patients diagnosed with sagittal synostosis were treated with skull reconstruction, including 11 males and 7 females. The age ranged from 6 to 9 months, with an average age of $10.0 \pm 1.8$ months. The patients were divided into two groups, 10 of which were in group A undergoing traditional skull reconstruction, without preoperative computer simulation and biomechanical analysis, and the remaining 8 patients were in group B undergoing computer simulation and biomechanical analysis to plan the optimal operation plan before surgery, as shown in Table 1.

Table 1 Patient data statistics

\begin{tabular}{ccccc}
\hline Patient & \multicolumn{2}{c}{ A } & \multicolumn{2}{c}{ B } \\
& Sex & Age(month) & Sex & $\begin{array}{c}\text { Age } \\
\text { (month) }\end{array}$ \\
\hline 1 & male & 8 & male & 8 \\
2 & male & 11 & female & 10 \\
3 & male & 9 & male & 8 \\
4 & female & 8 & female & 10 \\
5 & female & 12 & female & 9 \\
6 & male & 13 & male & 11 \\
7 & female & 13 & male & 11 \\
8 & male & 12 & female & 13 \\
9 & male & 10 & - & - \\
10 & male & 9 & - & - \\
\hline
\end{tabular}

\subsection{Inclusion and exclusion criteria}

\subsubsection{Inclusion criteria}

Those meeting inclusion criteria were patients who were diagnosed as sagittal synostosis, who were suitable for skull reconstruction, whose whole body can withstand the operation without respiratory and circulatory dysfunction.

\subsubsection{Exclusion criteria}

Patients whose clinical data were not available or incomplete were excluded from this study.

\subsection{Research method}

\subsubsection{Acquisition of clinical data}

Craniosynostosis is usually diagnosed by cranial X-ray, CT scan, medical CT reconstruction, MRI, and prenatal ultrasound. Through the information center, image data of patients were obtained, and general information of patients was collected through the case borrowing system, including operation time, blood loss, hospitalization cost and complications. The preoperative and postoperative models of the enrolled patients were reconstructed using the MIMICS 17.0 (Mimics 17.0,
Materialise company)- a medical three-dimensional reconstruction software, and the preoperative and postoperative cephalic index (CI, normal $75-85 \%$ ), head circumference $(\mathrm{CC})$ and cranial asymmetry index (CVAI, when it exceeds $3.5 \%$, it is considered significantly asymmetric) were calculated. The cephalic index (CI) is equal to the percentage of the maximum transverse diameter of the skull to the maximum anteroposterior diameter of the skull. Cranial vault asymmetries index (CVAI) is the percentage of the difference between the lengths of two oblique paths angle by $30^{\circ}$ from the anterior and posterior poles of the skull to the shorter oblique path.

\subsubsection{Computer aided simulation of surgery and biomechanical analysis of the detailed process}

Computer aided simulation of surgery: First, the patient's head CT scan Dicom file is imported into the Mimics software, which will automatically capture the skull pixels according to the skull density threshold and realized 3D reconstruction of skull model. On this basis, the head circumference, skull index and skull asymmetry index of the skull model can be measured by software. At the same time, the skull model can be 3D printed to understand the specific structure of the diseased skull model, as shown in Fig.1.
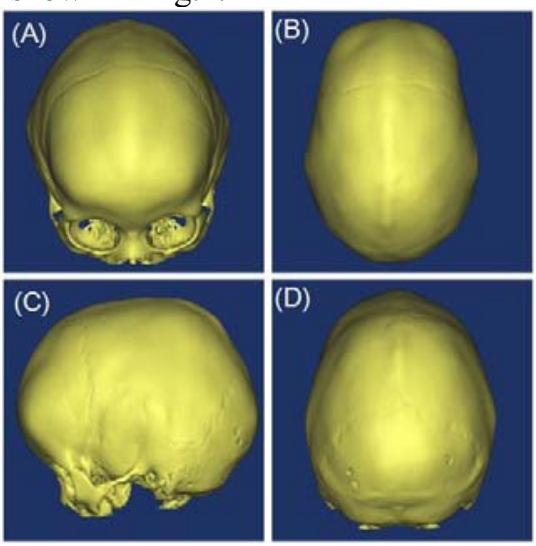

Fig 1 Skull model of a patient with sagittal synostosis after three-dimension reconstruction

Mimics software includes a whole set of surgical simulation system, which can perform skull cutting, flipping, splicing and so on to simulate the surgical process. Multiple surgical schemes can be simulated for the same patient, and the best individualized treatment scheme can be determined according to the calculated and measured cephalic index, head circumference, cranial asymmetry index and other data, as shown in figure 2. A variety of surgical procedures are simulated for the skull model of the patient shown in figure 1 . Then, based on the measured data, it can be concluded that the plan shown in figure 2 is the best individualized treatment plan for this patient. The specific treatment plan can be described as follow. Bone lamina 1 is obtained by cutting $1.5 \mathrm{~cm}$ from the superior orbital margin and $1 \mathrm{~cm}$ before the coronal suture, and then strip cuts are made above and below bone lamina 1 to make it fan out. After that, bone lamina 3 is obtained by cutting 
along the coronal suture by $1 \mathrm{~cm}$ and $2 \mathrm{~cm}$ respectively, and then the bone lamina 3 is removed along the skull base and finally placed behind the occipital to reconstruct the occipital node. Then, bone lamina 4 and 5 are obtained by cutting along the blue line. Bone lamina 2 is a coronal suture lamina, which is then cut in the middle line and opened laterally. Then lamina 4 is inserted into the middle seam of lamina 2 to reconstruct the normal skull frame. Lamina 5 is a floating bone lamina, which provides space for the brain to grow to both sides.
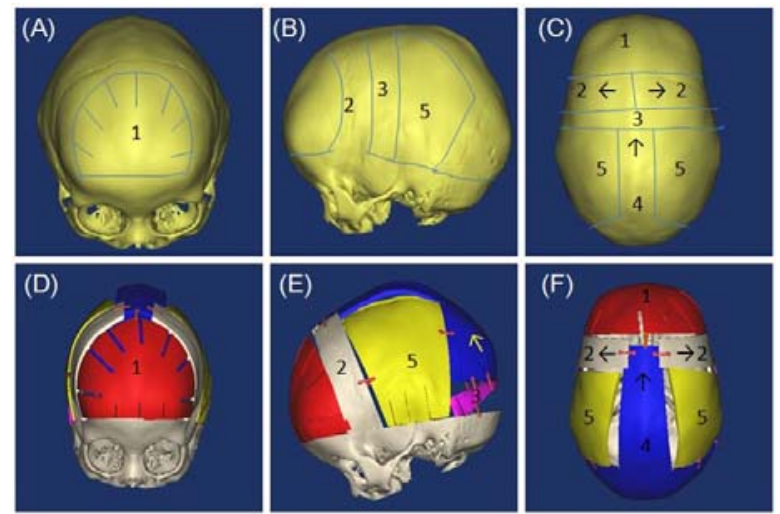

Figure 2. Optimal individualized treatment scheme for the same patient based on computer aided simulation

The first step, as shown in Fig.2, the brown sheet is the titanium sheet between the bone sheets. Based on previous surgical experience, the connecting splice is placed where bone splice may be required. The connecting splice between skulls is simulated by Boolean operation and the postoperative skull model is obtained. The second step, the files obtained by Mimics software are in STL format, which cannot be evaluated for additional properties and stresses. It is necessary to import STL format file into CATIA (CATIA V5) for materialization, and then export IGS format to Hypermesh (Hypermesh 13.0) software for finite element model analysis of the entire postoperative skull model. The finite element analysis can give the bone properties to the skull and the metal titanium properties to the connecting titanium sheets. The skull force is mainly the pressure perpendicular to the skull and the transverse force between the bones. By preloading the additive attribute, the stress and strain nephograms are obtained, as shown in Fig.4. It can be seen from Figure 4 that the color gradually changes from blue to red, indicating that the stress gradually increases. The forces in the yellow and green areas are between the maximum force and minimum force. The skull base is a single uncut bone, which will not be deformed even under heavy load. Due to the reassembly of cranium, the stress is mainly concentrated in the area contacting skull and in the joint area of titanium sheet. The red area is where the forces are most concentrated. The third step, in figure 4, the position where the titanium sheet is placed and turns red is the position with the greatest stress, and titanium sheets must be placed in this position during the operation. In step 1 , the position where the titanium sheets are placed but does not turn red is the position where no placement of titanium sheets is needed, so this position is removed. In the place where the titanium sheets need to be placed but have not been placed in advance, obvious deformation will occur under the additional load, so the titanium sheets need to be added in this position. Repeat the cycle from the first step to the third step until the skull force is perfectly supported and no more deformation occurs. At this point, the placement position and number of fixed objects obtained are the best. As shown in figure 4, 13 titanium sheets are needed to perfectly support the skull in accordance with the surgical plan in Figure 2 after stress calculation.
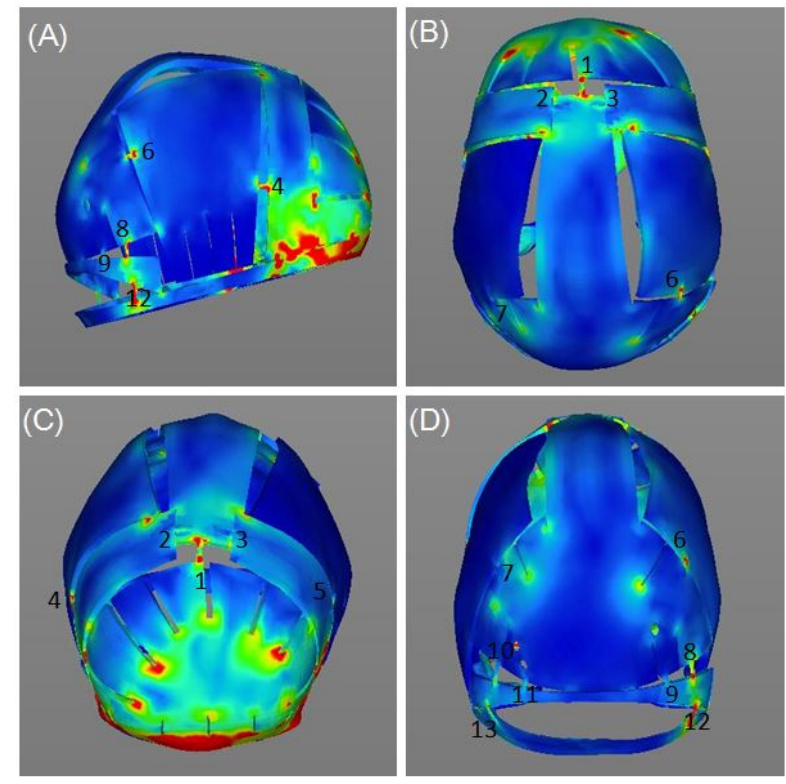

Fig. 3-D Cloud diagram of finite element stress calculation: It can be seen from Figure 3 that the color gradually changes from blue to red, indicating that the stress gradually increases. The forces in the yellow and green areas are between the maximum force and minimum force. The skull base is a single uncut bone, which will not be deformed even under heavy load. Due to the reassembly of cranium, the stress is mainly concentrated in the area contacting skull and in the joint area of titanium sheet. The red area is where the forces are most concentrated. In figure 3, the position where the titanium sheet is placed and turns red is the position with the greatest stress, and titanium sheets must be placed in this position during the operation. The number indicates the position and number of titanium sheets. A total of 13 titanium sheets are required.

\subsection{Conducting operation according to the preoperative simulation plan}

Neurosurgeons only need to cut the skull according to the simulated preoperative surgical plan, and place the titanium sheets in the optimal position, which significantly reduces the operation time and bleeding. No skull pieces were deformed or broken when suturing the skin, as shown in Figure 4. Postoperative CT review is basically consistent with the preset program, as shown in Figure 5. 


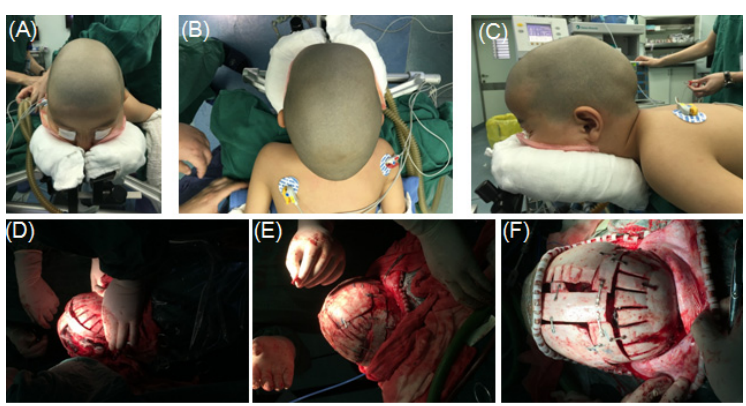

Figure 4. implementing surgical procedures according to the preset program
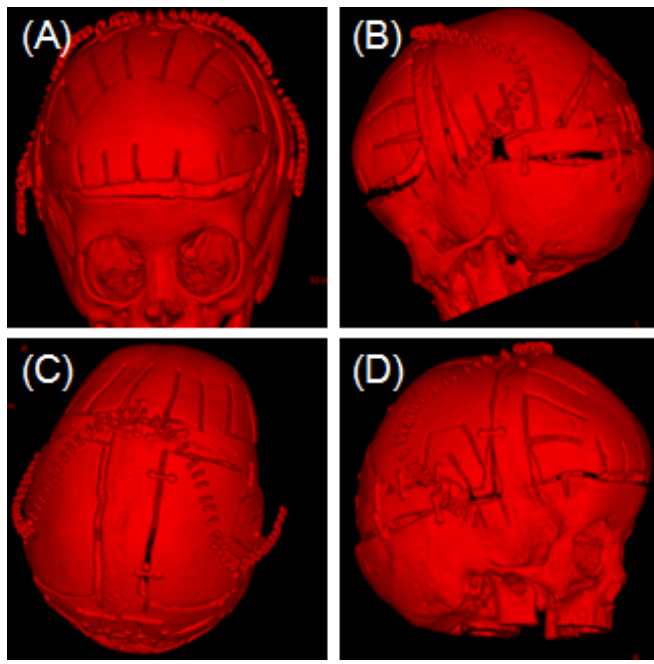

Figure 5. Postoperative 3D CT reconstruction

\subsection{Statistical analysis}

SPSS (IBM SPSS Statistics 22) software was used for statistical analysis. Heterogeneity between the two groups was tested by the Mann-Whitney test (test criterion is $\mathrm{P}<0.05$ ). Wilcoxon signed-rank test (test criterion is $\mathrm{P}<0.05$ ) was used for comparison of preoperative and postoperative numerical changes.

\section{Results}

The average operation time of group A was $276 \pm 23.5$ min, and that of group B was $217 \pm 29.3 \mathrm{~min}$, which was significantly shorter than that of group $\mathrm{A}$ and the difference was statistically significant $\left(P_{\text {time }}<0.001<\right.$ 0.05 according to the Mann-Whitney test, IBM SPSS Statistics 22.0) . The average operative blood loss in group A was $90 \pm 15.5 \mathrm{~mL}$, and that in group B was $70 \pm$ $11.7 \mathrm{~mL}$, which was less than that in group $\mathrm{A}$ and the difference was statistically significant $\left(P_{\text {blood }}=0.009<\right.$ 0.05 according to the Mann-Whitney test ) . The average surgical cost of group A was $34495 \pm 8662 ¥$, and that of group B was $25149 \pm 4133 ¥$, so the difference was statistically significant $\left(P_{\text {cost }}=0.004<\right.$ 0.05 according to the Mann-Whitney test) . In group A, 1 patient developed central system infection after surgery and improved after treatment; while in group B, no obvious postoperative complications occurred, as shown in Table 2 and Table 3.

The average cephalic index of group A before surgery was $69 \pm 2.3 \%$, and the average postoperative cephalic index was $80.5 \pm 3.9 \%$, indicating that the cephalic index was significantly improved $\left(P_{\mathrm{CI}(\mathrm{A})}=0.005\right.$ $<0.05$, according to the Wilcoxon signed-rank test). The cephalic index of group B was $67.5 \pm 2.4 \%$ before surgery and $80.5 \pm 3.7 \%$ after surgery, indicating that cephalic index of group B was significantly improved $\left(P_{\mathrm{CI}(\mathrm{B})}=0.012<0.05\right.$, according to the Wilcoxon signedrank test). There was no significant change in preoperative and postoperative head circumference for both group A and Group B. The cranial asymmetry index of group A was $2.2 \pm 0.9 \%$ before surgery and $3.6 \pm$ $0.6 \%$ after surgery, which indicates that the cranial asymmetry index in group A became more higher after surgery, and the difference was of statistical significance $\left(P_{\mathrm{CAVI}(\mathrm{A})}=0.005<0.05\right)$. The cranial asymmetry index of group B was $2.05 \pm 0.8 \%$ before surgery and $2.1 \pm$ $0.6 \%$ after surgery, indicating that the cranial asymmetry index of group B was low no matter before or after surgery, and the change was not statistically significant $\left(P_{\mathrm{CAVI}(\mathrm{B})}=0.887>0.05\right)$.

\begin{tabular}{|c|c|c|c|c|c|c|c|c|c|c|}
\hline \multirow[t]{3}{*}{ Patient } & \multicolumn{10}{|c|}{ Group A } \\
\hline & \multirow{2}{*}{$\begin{array}{l}\text { Operative } \\
\text { Time (min) }\end{array}$} & \multirow{2}{*}{$\begin{array}{l}\text { Blood Loss } \\
\text { Volume (ml) }\end{array}$} & \multirow{2}{*}{$\begin{array}{l}\text { Hospital } \\
\text { Cost(Y) }\end{array}$} & \multirow{2}{*}{$\begin{array}{l}\text { Complicatio } \\
\text { ns }\end{array}$} & \multicolumn{2}{|c|}{$\mathrm{CI}-(\%)$} & \multicolumn{2}{|c|}{ CC-(cm) } & \multicolumn{2}{|c|}{ CVAI-(\%) } \\
\hline & & & & & Pre- & Post- & Pre- & Post- & Pre- & Post- \\
\hline 1 & 252 & 120 & 31,524 & $\begin{array}{l}\text { central } \\
\text { nervous } \\
\text { system } \\
\text { infection }\end{array}$ & 68 & 78 & 48 & 46 & 3.4 & 3.6 \\
\hline 2 & 290 & 80 & 45,632 & none & 65 & 76 & 50 & 47 & 2.8 & 3.2 \\
\hline 3 & 248 & 90 & 29,358 & none & 70 & 84 & 45 & 47 & 2.4 & 4 \\
\hline 4 & 313 & 80 & 34,536 & none & 72 & 83 & 49 & 46 & 1 & 2.6 \\
\hline 5 & 275 & 100 & 41,348 & none & 69 & 74 & 45 & 48 & 0.8 & 2.4 \\
\hline 6 & 301 & 70 & 34,453 & none & 66 & 73 & 47 & 51 & 1.2 & 4 \\
\hline 7 & 293 & 80 & 32,132 & none & 70 & 82 & 46 & 47 & 2 & 3.6 \\
\hline 8 & 236 & 110 & 40,781 & none & 67 & 80 & 42 & 45 & 1.3 & 2.8 \\
\hline 9 & 268 & 90 & 25,784 & none & 69 & 81 & 46 & 47 & 2.7 & 3.7 \\
\hline 10 & 277 & 110 & 56,821 & none & 72 & 83 & 48 & 46 & 2.4 & 4.2 \\
\hline \multirow[t]{2}{*}{ median } & $276 \pm 23.5$ & $90 \pm 15.5$ & $\begin{array}{l}34,495 \pm \\
8,662\end{array}$ & & $69 \pm 2.3$ & $80.5 \pm 3.9$ & & & $2.2 \pm 0.9$ & $3.6 \pm 0.6$ \\
\hline & & & & & \multicolumn{2}{|c|}{$P=0.005$} & \multicolumn{2}{|c|}{$P=0.643$} & \multicolumn{2}{|c|}{$P=0.005$} \\
\hline
\end{tabular}




\begin{tabular}{|c|c|c|c|c|c|c|c|c|c|c|}
\hline \multicolumn{9}{|c|}{ Table 3. Participants and Statistical Data } & & \\
\hline \multirow{3}{*}{ Patient } & \multicolumn{10}{|c|}{ Group B } \\
\hline & \multirow{2}{*}{$\begin{array}{c}\text { Operative } \\
\text { Duration } \\
(\min )\end{array}$} & \multirow{2}{*}{$\begin{array}{l}\text { Blood Loss } \\
\text { Volume (ml) }\end{array}$} & \multirow{2}{*}{$\begin{array}{l}\text { Hospital } \\
\text { Cost(Y) }\end{array}$} & \multirow{2}{*}{$\begin{array}{l}\text { Complicatio } \\
\mathrm{n}\end{array}$} & \multicolumn{2}{|c|}{$\mathrm{CI}(\%)$} & \multicolumn{2}{|c|}{$\mathrm{CC}(\mathrm{cm})$} & \multicolumn{2}{|c|}{ CVAI (\%) } \\
\hline & & & & & Pre- & Post- & Pre- & Post- & Pre- & Post- \\
\hline 1 & 182 & 80 & 23,578 & none & 67 & 78 & 47 & 50 & 2.4 & 2.2 \\
\hline 2 & 230 & 70 & 30,145 & none & 66 & 76 & 49 & 47 & 3.2 & 3 \\
\hline 3 & 242 & 80 & 24,312 & none & 71 & 83 & 46 & 45 & 1.2 & 1.4 \\
\hline 4 & 164 & 60 & 23,128 & none & 70 & 83 & 48 & 45 & 2 & 1.8 \\
\hline 5 & 175 & 70 & 25,986 & none & 66 & 75 & 45 & 48 & 2.1 & 1.7 \\
\hline 6 & 204 & 50 & 30,817 & none & 65 & 77 & 45 & 51 & 3.5 & 3.2 \\
\hline 7 & 230 & 70 & 20,345 & none & 71 & 84 & 46 & 49 & 1.6 & 2 \\
\hline 8 & 240 & 90 & 32,981 & none & 68 & 83 & 45 & 49 & 1.8 & 2.4 \\
\hline \multirow[t]{2}{*}{ median } & $217 \pm 29.3$ & $70 \pm 11.7$ & $\begin{array}{l}25,149 \pm \\
4,133\end{array}$ & & $67.5 \pm 2.4$ & $80.5 \pm 3.7$ & & & $2.05 \pm 0.8$ & $2.1 \pm 0.6$ \\
\hline & & & & & \multicolumn{2}{|l|}{$P=0.012$} & \multicolumn{2}{|c|}{$P=0.137$} & \multicolumn{2}{|l|}{$P=0.887$} \\
\hline
\end{tabular}

\section{Discussion}

Sagittal synostosis requires immediate surgery as indicated by increased cranial pressure, mental retardation, impaired or absent vision, cranial deformity, and mental and spiritual defects. However, it is sometimes difficult clinically to distinguish between skull deformity due to premature closure of the craniosynostosis and skull deformity due to delayed brain development. Plain CT scan and 3D CT reconstruction of skull can provide references for differential diagnosis. Even though the patient's medical history and clinical manifestation can serve as the basis for diagnosis, the final diagnosis of craniosynostosis still depends largely on CT[6-8].

For the exploration and research on the treatment of craniosynostosis, the head of human body or sheep body was used as material for teaching and simulating surgery at early stage[9-10]. However, these materials are difficult to obtain, and the collection and storage of these materials requires special equipment.

At present, in the field of head and neck surgery, the advantages of pre-surgical simulation are already apparent. Accordingly, a number of surgical simulation softwares have been developed for clinical use. In the early 21st century, Mommaerts[11] published an paper on the application of software to simulate the treatment of craniosynostosis, in which it was described that the software could cut and flip the virtual skull. Rodt[12] found that a patient with premature closure of frontal suture achieved good operation results through preoperative software simulation. Hwang[13] published an paper on the treatment of craniosynostosis by digital assistant design, in which doctors can choose the best surgical plan, thus saving time for intraoperative thinking.

With the development and popularization of $3 \mathrm{D}$ printing technology, there have been reports on various types of surgical stents, positioning of bone segment in $\mathrm{CAD} / \mathrm{CAM}$ design and the guidance of osteotomy [1416]. Danelson et al. [17] used a computer-aided design program to design a support structure, and then printed a 3-D skull model to facilitate the design of a personalized spring brace system for skull reconstruction. Meehan et al. [18] also verified the application value of computer- aided simulation surgery in craniofacial surgery and in treatment of craniosynostosis. Flores et al. [19] used Maya 8.5(Autodesk, San Rafael, CA, USA) to make virtual 3D head models for clinical teaching and atlas surgery. Mardini[20] used computer-aided manufacturing techniques to create prefabricated templates and made plans prior to craniosynostosis osteotomy. These computer-aided methods can reflect the anatomy of each patient and are easier to use than biological models. When doctors know in advance how to cut, it can make the surgical procedure easier and reduce blood loss [12], thus customizing and optimizing treatment schemes for patients. We aim to determine the accuracy of the execution process during osteotomy and implant fixation, and to achieve the best skull shape and cranial volume by operating computer-aided simulation techniques.

Computer aided simulation plays an important role in making the operation plan. Having a complete set of operation plan not only reduces the operation time, but also reduces intraoperative blood loss and reduces the chance of blood transfusion. In the virtual operating system, the computer reconstruction model accuracy can achieve anatomical accuracy, so we can visually observe and evaluate the area to be operated, so as to achieve the optimal cranial cavity expansion and obtain bone flap location. Prior to osteotomy operation, the simulation of skull reconstruction can enable the surgeon to fully understand the characteristics of the patient's reconstructed skull and know the operation process very well. This certainly saves time thinking about cutting and moving the bone intraoperatively. Through the computer virtual simulation operation, it can provide surgeons with a good experience and understanding of the process.

Although 3D computer simulation surgery has been reported, this paper for the first time shows that preoperative computer simulated stress calculation can achieve aesthetic effect of the operation, reduces the surgical consumables and decreases operation cost. In conclusion, the optimal surgical effect was achieved by preoperative stress calculation. Due to the uneven thickness and complex shape of cranium, so the biomechanical analysis of cranium is a major challenge. However, in skull reconstruction for treatment of craniosynostosis, biomechanical calculation is very necessary. Empirically placing a fixator without 
biomechanical calculation will not only increase the number of fixators, but also lead to prolonged operation time, high operation cost, unattractive skull shape, and increased risk of postoperative infection. If the number of the fixators is insufficient or the placement location is not accurate, the most serious consequence may be postoperative deformation due to the imperfect support between the reconstructed bone plates. In a few cases, after scalp suture, deformity of reconstructed skull and fracture of joint will even occur under the effect of scalp load, which makes the surgeon in an awkward situation and has to repull the scalp and fix it again, thus further extending the operation time.

Bone is a living organ with perfect mechanical support, which can avoid fracture, collapse and fracture in the near future and make the skull grow symmetrically in the long term. Uneven mechanical load will lead to the apoptosis of bone-related cells, which will lead to the decrease of bone quality and quantity, and then lead to the loss of reconstructed bone plate's function of evenly dispersing stress around, so that the stress transferred to the edge of the bone plate cannot be further transferred around effectively and uniformly. Therefore, a perfect cranial mechanical support is very important for even and symmetrical growth of skull after surgical reconstruction. The operation plan after biomechanical calculation can not only ensure the beautiful and symmetrical skull shape, but also ensure the normal growth of skull bones in the long term, and achieve better growth effect after bone suture healing. Therefore, biomechanical calculation after skull reconstruction is a critical process.

It is very difficult for the computer simulation and attribute addition of skull due to the variability of skull structure, the multiple radian directions of skull and the diversity of skull nature. Therefore, how to effectively solve the geometric model simulation and attribute addition has become a difficult problem in the research of mechanics after skull reconstruction. Only by solving these problems well can we carry out effective finite element simulation and mechanical analysis. The emergence of Mimics software for medical processing, the advent of high-precision CT images and the rapid development of digital science and technology make stress bioanalysis possible. Finite Element Method (FEM) is an important method of digital biomechanics analysis. Currently, the biomechanics represented by FEM is increasingly used in multiple medical fields. Finite element analysis can be used to calculate the stress and strain of the reconstructed skull model under different external forces.

Skull reconstruction requires a fixed material, i.e. titanium sheets, but it is difficult to know where to place the titanium sheets and how many titanium sheets are needed to achieve perfect mechanical support. Therefore, it is important to have reliable simulated surgical results, based on which we can determine the number of titanium sheets required and the placement location of the titanium plates.

These fixed materials are often expensive. As a foreign body, the fixation material may delay wound healing. Using fewer foreign materials can help reduce the risk of infection. Some foreign bodies can act as good culture medium leading to pyogenic infections. Infection of the central nervous system can sometimes be fatal in children. Preoperative biomechanical analysis can avoid overuse of repair materials. Force analysis allows the skull to be symmetrical, giving the head a better appearance. In conclusion, biomechanical analysis enables each patient's reconstructed skull to achieve perfect mechanical support. This study proposes a biomechanical system to assist surgeons in improving the accuracy, safety, and effectiveness of surgery.

\section{Conclusion}

Sagittal synostosis is the most common type of craniosynostosis. In the treatment of sagittal craniosynostosis, computer-aided simulation surgery and biomechanical analysis enable surgeons to have a clear understanding of the operation process before surgery. Although 3D computer simulation surgery has been reported, this paper for the first time shows that preoperative computer simulated stress calculation can achieve aesthetic effect of the operation, reduces the surgical consumables and decreases operation cost. In conclusion, the optimal surgical effect was achieved by preoperative stress calculation. Most importantly, compared with traditional surgery, the new treatment method reduces the operation time, blood loss and operation cost.

\section{Acknowledgment}

This study is supported by Key Project of International Cooperation on Science and Technology Innovation Among Governments of National Key Research and Development Plan (2017YFE0121200)

Li Xiang and Lu Zheng contributed equally as first authors

\section{References}

1. C.S. Mendoza, N. Safdar, K. Okada, E. Myers, G.F. Rogers, M.G. Linguraru. Med Image Anal, 18:635646 (2014)

2. M. Engel, C. Freudlsperger, J. Hoffmann, J. Mühling, G. Castrillon-Oberndorfer, R. Seeberger. J Craniomaxillofac Surg, 40:363-368 (2012)

3. J. Barritt, M. Brooksbank, D. Simpson. Dev Med Child Neurol, 23:183-191 (1981)

4. D.G. Vollmer, J.J. Jane, T.S. Park, J.A. Persing. J Neurosurg, 61:557-562 (1984)

5. J. Gateno, J.F. Teichgraeber, E. Aguilar. Plast Reconstr Surg, 105:873-882 (2000)

6. A. Taccone, A. Cama, M. Ghiorzi, M.P. Fondelli. J. Radiol Med, 77(4):322-328 (1989)

7. W.M. Vannier, C.F Hildebolt, J.L. Marsh, T.K. Pilgram, W.H. McAlister, G.D. Shackelford. J. Radiology, 173(3):669-673 (1989)

8. T.K. Pilgram, M.W. Vannier, C.F. Hildebolt, J.L. Marsh, W.H. McAlister, G.D. Shackelford, C.J. 
Offutt, R.H. Knapp. Radiology, 173(3):675-679 (1989)

9. E. Aboud, O. Al-Mefty, M.G. Yasargil. New laboratory model for neurosurgical training that simulates live surgery. J Neurosurg, 97:1367-1372 (2002)

10. T. Hicdonmez, T. Parsak, S. Cobanoglu. J Neurosurg, 105:150-152 (2006)

11. M.Y. Mommaerts, G. Jans, S.J Vander, P.F Staels , G. Van der Perre, R. Gobin. J Craniofac Surg, 12(6):547-554 (2001)

12. K. Imai, K. Tsujiguchi, C. Toda, E. Enoki, K.C. Sung, H. Sakamoto, S. Kitano, M. Hatoko, S. Tajima. Neurol Med Chir (Tokyo), 39(6):423-426, 427 (1999)

13. H.S Hwang, C.H Hwang, K.H Lee, B.C Kang. Am J Orthod Dentofacial Orthop, 130(6):779-785 (2006)

14. M.J. Zinser, H.F. Sailer, L. Ritter, B. Baumann, M. Maegele, J.E. Zoller. J Oral Maxillofac Surg, 71:2151, e1-2151.e21 (2013)

15. M.J. Zinser, R.A. Mischkowski, H.F Sailer, J.E Zoller. Oral Surg Oral Med Oral Pathol Oral Radiol, 113:673-687 (2012)

16. Li. B, Zhang. L, Sun. H, Yuan. J, Shen. SGF, Wang X. Br J Oral Maxillofac Surg, 51:e239-e244 (2013)

17. K.A. Danelson, E.S. Gordon, L.R. David, J.D. Stitzel. Biomed Sci Instrum, 45:358-363 (2009)

18. M. Meehan, M. Teschner, S. Girod. Orthod Craniofac Res, 6(Suppl 1):102-107 (2003)

19. R.L. Flores, N. Deluccia, B.H. Grayson, A. Oliker, J.G. McCarthy. Plast Reconstr Surg, 126:2084-2092 (2010)

20. S. Mardini, S. Alsubaie, C. Cayci, H. Chim, N. Wetjen. J Plast Reconstr Aesthet Surg, 67:336-343 (2014) 